\title{
Application Research on the Maintainability Allocation Method of a Certain Shooter Seat
}

\author{
Liying Jin $\mathbb{D}^{1,2}$ Wensheng Wang, ${ }^{3,4}$ HouYong Shu, ${ }^{4}$ Xuemei Ma, ${ }^{4}$ Chenxing Liang, ${ }^{5}$ \\ and Qiang $\mathrm{Ma}^{2}$ \\ ${ }^{1}$ Xiangyang Key Laboratory of Intelligent Manufacturing and Machine Vision, Hubei University of Arts and Science, \\ Xiangyang 441053, China \\ ${ }^{3}$ Aviation Key Laboratory Science and Technology on Life-Support Technology, Hubei, Xiangyang 441003, China \\ ${ }^{4}$ Aerospace Life-Support Industries, Ltd., Hubei, Xiangyang 441003, China \\ ${ }^{5}$ School of Energy and Power Engineering, Xi'an Jiaotong University, Xi'an, Shaanxi 710049, China \\ ${ }^{2}$ School of Automotive and Transportation Engineering, Hubei University of Arts and Science, Xiangyang 441053, China
}

Correspondence should be addressed to Liying Jin; ggnjinliying@stu.xjtu.edu.cn

Received 23 August 2021; Accepted 3 December 2021; Published 29 December 2021

Academic Editor: Jian Shi

Copyright (c) 2021 Liying Jin et al. This is an open access article distributed under the Creative Commons Attribution License, which permits unrestricted use, distribution, and reproduction in any medium, provided the original work is properly cited.

In view of the traditional maintainability allocation method for a certain shooter seat for maintainability allocation did not consider the lifecycle expense problem, the improved NSGA-II algorithm (iNSGA-II, for short) is adopted to establish a multiobjective comprehensive trade-off model for a certain shooter seat product lifecycle maintenance-related expenses and mean time to repair (MTTR, for short) and construct multiobjective optimization problem. The experimental results show that the Pareto optimal solution effectively solves the limitation of the traditional maintainability allocation method and then provides a basis for a certain shooter seat to obtain a reasonable maintainability allocation scheme. The superiority of the iNSGA-II algorithm to optimize the maintainability allocation of a certain shooter seat was verified by comparing it with the traditional maintainability allocation method.

\section{Introduction}

In the specified conditions and time, according to the specified procedures and methods to repair the product, so that it can return to the state of the state or maintain the original ability, this process is called maintainability $[1,2]$. According to the information provided, one-third of the operating costs of large mechanical systems are used for maintenance, and one-third of the employees are required to perform maintenance services. If these are included, the total maintenance costs are more than 10 times the equipment costs. The information provided shows that it is not easy to repair equipment, so new ones are bought instead. However, the equipment maintenance cost is often more than the lab's afford, so the investment to new equipment is wasted. According to the results of the US study, they invested US $\$ 1$ in the development of the machine to improve the maintainability and in the future may get the benefit of reducing the lifecycle cost of US $\$ 50$ to US $\$ 100$.

Maintainability engineering began in the 1990s with the goal of reducing labor and maintenance costs to improve the operational effectiveness of military equipment [3-6]. Maintainability engineering not only affects the performance of the product but also affects the combat capability and logistics support of the troops. It runs through all stages of the lifecycle process of product demonstration, verification, production, use, decommissioning, and so on [7]. However, the system of modern military equipment is becoming more and more complex, which poses many problems for the maintenance design work. Maintenance allocation is the core work of the system or product for maintenance design. It runs through every stage of the system design, and the reasonable maintenance allocation 
method can solve the above problems. The application in the aircraft field is mainly explained in two aspects [8]:

(1) Provide maintenance design requirements to the designer of the product on the basis of ensuring that the product meets the maintainability requirements specified in the agreement

(2) Through the maintenance allocation, the maintenance indicators of each component are clarified so as to determine the maintainability requirements of the supporting products, and it is convenient for the contractor to implement the management products

In this paper, we introduce a new design approach for the maintainability allocation method to solve the limitations of the traditional maintainability allocation method. The novelty and the contributions are as follows:

(1) In the case of maintenance allocation, determine the reasonable and effective maintenance allocation plan according to the maintainability requirements of the product. Establishing a multiobjective comprehensive trade-off model for a certain shooter seat product lifecycle maintenance-related expenses and mean time to repair, the maintainability allocation problem is described as a multiobjective optimization problem.

(2) Considering that the limitations of the traditional maintainability allocation method, this paper used an improved NSGA-II algorithm (iNSGA-II, for short) to solve the maintainability allocation problem of a certain shooter seat and obtain the optimal Pareto solution.

(3) The superiority of the iNSGA-II algorithm to optimize the maintainability allocation of a certain shooter seat was verified by comparing it with the traditional maintainability allocation method.

The rest of this paper is organized as follows. Section 2 presents the design and multiobjective comprehensive tradeoff model of a certain shooter seat. Section 3 introduces the iNSGA-II algorithm. Section 4 presents the application of the iNSGA-II in a certain shooter seat. The paper ends with Section 5 where a conclusion is made.

\section{Establishment of Comprehensive Trade-Off Model for Maintainability Allocation}

The National Military Standard lists five commonly used methods in the Maintainability Allocation and Estimation Manual [1]:

(1) Equivalence allocation method

(2) Failure rate allocation method

(3) Comprehensive weighting factor allocation method according to failure rate and design characteristics

(4) Method of allocation based on data for repairing similar products

(5) Weighted allocation method to ensure availability and consideration of differences in the complexity of each unit
The five commonly used maintainability allocation methods only consider the maintainability time, without considering the design and maintenance costs of the system. According to the requirements of "full cost management" and "pursuing the highest cost-performance ratio," the design ideas of these five methods do not conform to the design concept of the new equipment. For example, in the process of maintainability design, if the MTTR left for a maintenance unit is not sufficient, this commonly used method is difficult to be completed, and other methods must be used, which will increase the difficulty of the design and also increase the manufacturing cost.

Maintainability allocation [9] can usually be regarded as a multiobjective optimization problem with the objective function of product lifecycle maintenance-related expenses and MTTR. The description of the maintainability allocation problem as a multiobjective optimization problem has the following advantages:

(1) By considering the product's lifecycle maintenancerelated expenses and MTTR as separate entities, no preference needs to be introduced in the search process

(2) Considering the lifecycle maintenance-related expenses and MTTR at the same time, rather than giving priority to the MTTR in isolation, it will be more conducive to give a reasonable maintainability allocation scheme

2.1. Comprehensive Weighting Factor Allocation Method according to Failure Rate and Design Characteristics. In engineering practice, the comprehensive weighting factor allocation method according to failure rate and design characteristics is the most commonly used traditional maintainability allocation method. This method converts the factors (complexity, testability, reachability, replaceability, adjustability, maintenance environment, etc.) considered in the allocation into weighting factors, which are allocated according to the weighting factors of design characteristics. The line replaceable unit (LRU, for short) of MTTR is as follows:

$$
\operatorname{Min} J_{c c t}=\beta_{i} \times \bar{M}_{c t} \text {, }
$$

where $\bar{M}_{c t}$ is system mean time to repair and $\beta_{i}$ is the comprehensive weighting coefficient of the repair time.

$$
\begin{aligned}
& \beta_{i}=\frac{k_{i}}{\bar{k}} \times \frac{\bar{\lambda}}{\lambda_{i}}, \\
& \bar{k}=\frac{\left(\sum_{i=1}^{n} k_{i}\right)}{n},
\end{aligned}
$$

where $\bar{\lambda}$ is the average failure rate of each unit of the system, $\lambda_{i}$ the failure rate of $i$-th unit, $\bar{k}$ is the average weighting factor of each unit of the system, and $k_{i}$ is the maintainability weighting factor of unit $i$-th, which is related to the product's fault detection and isolation method, accessibility, replaceable, and adjustability factors. The worse the maintainability, the larger $k_{i}$ :

$$
k_{i}=\sum_{j=1}^{m} k_{i j},
$$


where $k_{i j}$ is the weighting factor of the $i$-th unit and $j$-th factor, and $j=1,2, \ldots, m$ is the number of weighting factors. Their values are shown in table 1.

2.2. Research on New Maintainability Allocation Method. In order to make the allocation result accord with the engineering practice, the method of weakening the importance of the failure rate is adopted in the comprehensive weighting factor allocation method according to failure rate and design characteristics.

Let $a_{1 i}=\bar{\lambda} / \lambda_{i}$ indicate the degree of influence of failure rate on maintainability allocation and $a_{2 i}=k_{i} / \bar{k}$ represents the impact of product maintenance complexity on maintainability allocation. The comprehensive weighting coefficient $\beta_{i}$ can be expressed as follows:

$$
\beta_{i}=\alpha_{1 i} \times \alpha_{2 i}
$$

It can be seen from formula (5) that in the comprehensive weighting coefficient, the failure rate and the complexity of maintenance are equally important, but when the failure rates are quite different, that is, $a_{1 i}$ is very different, in contrast and $a_{2 i}$ is not very large, which will inevitably cause $\beta_{i}$ to be greatly affected by the failure rate. In engineering practice, although the LRU failure rates of the products are very different, only the replacement of the LRU is considered during the field maintenance, so the actual maintenance time is not much different.

Therefore, it is necessary to control the value of $a_{1 i}$. The logarithmic function is used to determine the value of $a_{1 i}$ by looking up relevant data, mainly because (1) the logarithmic function is a monotonic function in its domain, (2) taking the logarithm can convert multiplication to addition, and (3) after taking the logarithm, the property and correlation of the data will not be changed, but the scale of the variables is compressed to make the data more stable.

The logarithm of formula (2) is taken to get

$$
\begin{aligned}
\log _{\alpha} \beta_{i} & =\log _{\alpha} \frac{k_{\mathrm{i}} \times \bar{\lambda}}{\bar{k} \times \lambda_{i}} \\
& =\log _{\alpha} \frac{k_{\mathrm{i}}}{\bar{k}}-\log _{\alpha} \frac{\lambda_{i}}{\bar{\lambda}} \quad 0<\alpha<1 .
\end{aligned}
$$

In order to emphasize and maintain the impact of the design characteristics of the product, let: $\beta_{i}=\log a \beta_{i}$ and $\left(k_{i} / \bar{k}\right)$ instead of $\log a\left(k_{i} / \bar{k}\right)$; then formula (6) becomes

$$
\beta_{i}=\frac{k_{i}}{\bar{k}}-\log _{\alpha} \frac{\lambda_{i}}{\bar{\lambda}} \quad 0<\alpha<1 .
$$

Formula (7) is a method to weaken the importance of the failure rate based on the comprehensive weighting factor allocation method according to failure rate and design characteristics.

\subsection{A Multiobjective Comprehensive Trade-Off Model.} Typically, the trade-off model for maintainability allocation consists of a time model and expense model [3]. The main purpose of the time model is to establish the functional relationship between the system MTTR and the constituent units MTTR and to analyze the maintainability parameters and $M_{i}$ boundary conditions of the system and the constituent units. The expense model considers $M_{i}$ as the influencing factor of the cost so as to decompose the total cost of the system. Then, the cost of each maintenance unit can be described by $M_{i}$. According to foreign statistics and reference to the maintenance optimization allocation method, a comprehensive tradeoff model that considers the product's lifecycle maintenancerelated expenses $\left(J_{1}\right)$ and $\operatorname{MTTR}\left(J_{2}\right)$ :

$$
\begin{aligned}
\operatorname{Min}_{1}= & \sum_{i=1}^{n} k_{i} \times\left[\frac{1}{\left(\lambda_{i} \times M_{i}\right)}\right]^{\alpha i} \\
& +\lambda_{i} \times T_{i} \times\left(u_{i}+s_{i}\right) \times M_{i}, \\
J_{2}= & \sum_{i=1}^{n} \frac{\lambda_{i} \times \bar{M}_{i}}{\sum_{i=1}^{n} \lambda_{i}}, \\
\text { s.t. } \sum_{i=1}^{n}\left(u_{i}+s_{i}\right)= & S_{0}, \\
M_{i}^{l} \leq M_{i} \leq & M_{i}^{u},
\end{aligned}
$$

where $n$ is the number of product maintenance units; $k_{i}$ is the constant of the $i$-th maintenance unit greater than zero, depending on the maintenance influencing factors considered; $\lambda_{i}$ is the failure rate of the $i$-th maintenance unit; $a_{I}$ is the power index of the $i$-th maintenance unit, mainly determined by the structure of the maintenance unit and actual statistical data; $M_{i}$ is the boundary condition of the $i$-th maintenance unit; $T_{i}$ is the average service life of the $i$-th maintenance unit; $u_{i}$ is the unit time cost of maintenance repair for the $i$-th maintenance unit; $s_{i}$ is the loss of production per unit time of the product of the $i$-th maintenance unit; $S_{0}$ is the limit value of the maintenancerelated expenses of a system throughout the lifecycle; $M_{i}^{l}$ is the minimum maintenance time at the base level of the $i$-th maintenance unit; $M_{i}^{u}$ is maximum maintenance time allowed for base-level maintenance of the $i$-th maintenance unit; and $\bar{M}_{i}$ is MTTR of the $i$-th maintenance unit.

The mathematical model of product maintainability allocation is based on the minimization of equations (8) and (9). The MTTR of each maintenance unit of the product and the lifecycle maintenance-related expenses of the product are the constraints. The optimal maintainability allocation scheme can be obtained by selecting a reasonable algorithm according to the model.

The solution to the multiobjective optimization problem is usually a set of optimal solutions [10]. There is no uniqueness of the solution. The elements in the set of optimal solutions are called Pareto optimal. Pareto optimality is actually a set of equilibrium solutions, rather than a single global optimal solution [11].

\section{Improved NSGA-II Algorithm}

There are many ways to improve the clustering algorithm [12-15]. The most common algorithm for solving multiobjective optimization problems is nondominated sorting 
TABle 1: The value of maintainability weighting factor.

\begin{tabular}{|c|c|c|c|}
\hline Weighting factor & Type & $\begin{array}{c}k_{i j} \\
\text { value }\end{array}$ & Description \\
\hline \multirow{4}{*}{$\begin{array}{l}\text { Fault detection and isolation } \\
\text { factor, } k_{i 1}\end{array}$} & Automatic & 1 & Use the computer inside the device to detect the fault \\
\hline & Semiautomatic & 3 & Fault location is performed by detecting circuit in manual control machine \\
\hline & Artificial & 5 & Detect the detection hole set inside the machine with a portable instrument \\
\hline & Artificial & 10 & $\begin{array}{c}\text { There is no set detection hole in the machine, so it must be manually traced point } \\
\text { by point }\end{array}$ \\
\hline \multirow{4}{*}{ Accessibility factor, $k_{i 2}$} & Good & 1 & No need to remove the cover when replacing the failed unit \\
\hline & Better & 2 & Quickly remove covers \\
\hline & Difference & 4 & $\begin{array}{l}\text { Remove the block; tightening on loosening bolt is required to remove barrier and } \\
\text { cover }\end{array}$ \\
\hline & Very poor & 8 & $\begin{array}{l}\text { In addition to the upper and lower screws, more than two people are required to } \\
\text { move the obstruction and cover }\end{array}$ \\
\hline \multirow{4}{*}{ Replaceable factor $k_{i 3}$} & Plug & 1 & Replaceable unit is plug-in \\
\hline & Buckle & 2 & Replaceable modular module, open the buckle when replacing \\
\hline & Screw & 4 & Replace the unit with upper and lower screws \\
\hline & Welding & 6 & The replacement shall be welded \\
\hline \multirow{3}{*}{ Adjustability factor, $k_{i 4}$} & Not adjusted & 1 & No adjustment is required after replacing a failed unit \\
\hline & Fine-tuning & 3 & Adjust using in-machine adjustment elements \\
\hline & Joint tuning & 5 & Must be joint tuning together with other circuits \\
\hline
\end{tabular}

genetic algorithm II (NSGA-II, for short) [16]. However, the mutation operator used is relatively weak, which limits the diversity of the population to some extent. In addition, the convergence speed of the NSGA-II algorithm still needs to be improved. Therefore, this paper uses the iNSGA-II algorithm to design an antiredundant mutation operator [17] and a forward comparison operation based on the NSGA-II algorithm. In the initialization phase, half of the individual populations are randomly generated, and the other half are generated based on the feature distribution information.

In reference [18], the superiority of the iNSGA-II algorithm has been verified, and the parameters of the switchedflux motors are optimized using this algorithm. In this paper, the iNSGA-II algorithm is used to solve the maintainability allocation problem of a certain shooter seat and obtain the optimal Pareto solution, which effectively solves the limitations of the traditional maintainability allocation method and then provides a basis for a certain shooter seat to obtain a reasonable maintainability allocation scheme.

3.1. Antiredundant Mutation Operator. The antiredundancy mutation operator has the advantages of high global search ability and population diversity. The following three steps are used to realize the antiredundancy mutation operator:

(a) An individual $x_{i}=\left(x_{i 1}, x_{i 2}, \ldots, x_{i M}\right)$, where $i=1,2$, $\ldots, N$, is randomly selected, and three gene positions are randomly selected from this individual, satisfying $a \leq b$ and $a \neq c$. The gene segment $\left\{x_{i a}, \ldots, x_{i b}\right\}$ is stored in the temporary array $T_{a b}$, and then the gene position $c$ is randomly selected from $T_{a b}$.

(b) The maximum absolute value of all correlation coefficients between $c_{r k}$ and other selected features is recorded as $p$ (see formula (10)). If gene position $x_{r}=1$, let $x_{r}=0$ with a probability of $p$; if $x_{r}=0$, let $x_{r}=1$ with a probability of $1-p$.

(c) The $\left\{x_{c}, \ldots, x_{c+b-a}\right\}$ is replaced with the $T_{a b}$.

$$
\left.\begin{array}{l}
p=\max \left\{c_{r k}, k=1,2, \ldots, M \& x_{k}=1\right\}, \\
c_{r k}=\frac{\sum_{i=1}^{N}\left(y_{i r}-\bar{a}_{n r}\right) \times\left(y_{i k}-\bar{a}_{n k}\right)}{\sqrt{\sum_{i-1}^{N}\left(y_{i r}-\bar{a}_{n r}\right)^{2}} \times \sqrt{\sum_{i-1}^{N}\left(y_{i k}-\bar{a}_{n k}\right)^{2}}},
\end{array}\right\}
$$

where $c_{r k}$ represents the correlation coefficient between the $r$-th and $k$-th features, $y_{i r}$ represents the value of the $i$-th sample in the $r$-th feature, and $a_{n r}$ represents the average value of all samples in the $r$-th feature. $M$ is the number of feature dimensions.

3.2. Forward Comparison Operation. When sorting among nondominated individuals, the forward comparison operation is designed to avoid redundant nondominated frontiers so that it has an acceleration effect on all test problems and greatly improves the running speed of multiobjective optimization.

Maintainability is the maintenance of the equipment according to the specified methods and procedures under the specified time and conditions, making the equipment easy to repair, improving the efficiency of the equipment system, and reducing the lifecycle expense of use. Maintainability allocation is an important part of maintainability engineering.

Before the running of the iNSGA-II algorithm to obtain Pareto optimal solution in solving the multiobjective optimization problem, the data set is preprocessed for standardization. The binary league is used to select individuals in the population. Supposing the crossover probability as 0.9 and the population size sample number as an even number $-z$ is the dimension of sample; when $z$ is an odd, the population size sample size is $3^{*} z+1$; when $z$ is even, the sample size of the population is $3^{*} z$, and the maximum 
number of iterations is 500 . The convergence and diversity of the iNSGA-II algorithm have been verified in the literature [18]. Its principle is shown in Figure 1.

\section{The Application of the iNSGA-II in Certain Shooter Seat}

Taking a certain shooter seat as an example, the genetic operator is designed by determining the model parameters, and the model is calculated to verify the feasibility of the maintainability allocation model.

4.1. Comprehensive Weighting Factor Allocation Results according to Failure Rate and Design Characteristics. According to the value requirements in Table 1, a maintainability allocation is made to a certain shooter seat, and the results are shown in Table 2 .

From the allocation results in Table 2, it can be seen that the MTTR of the seat cover is 16.5 times that of the seat body. Because the repair of a certain shooter seat in the field is mainly completed by replacing the LRU, the maintenance time difference between the LRUs should be small, so the allocation results in Table 2 are obviously inconsistent with the engineering practice. From the calculation formula of the comprehensive weighting factor allocation method according to failure rate and design characteristics, it can be seen that the size of the failure rate has the greatest impact on the final allocation. The larger the difference between the two LRU failure rates, the greater the difference between the two allocation results. This method is not suitable for situations where the difference between the failure rates of the LRUs is large.

4.2. The Application of the New Maintainability Allocation Method. According to engineering experience, numerical simulation, and the change of $f(x)=\log \alpha(x), 0<\alpha<1$ the principle of value determination for $\alpha$ is made. (see Figure 2), as shown in Table 3 . The ratio of the maximum value to the minimum value of the failure rate is expressed by $L$.

A new maintainability allocation method was used to reassign a certain shooter seat. From Table 2, the ratio between the maximum and minimum LRU failure rates of a certain shooter seat is: $L=28.965 / 1.2996 \approx 22$; then $a$ $=0.001$. The maintainability allocation results of each LRU of a certain shooter seat are shown in Table 4.

According to the allocation results in Table 4, the MTTR of a certain shooter seat is calculated as follows:

$$
\begin{aligned}
J & =\sum_{i=1}^{n} \frac{\lambda_{i} \times \bar{M}_{i}}{\sum_{i=1}^{n} \lambda_{i}} \\
& =\frac{28.965 \times 18.0+1.2996 \times 7.5+15.552 \times 15.0}{28.965+1.2996+15.552}
\end{aligned}
$$

$=16.7 \mathrm{~min}$.

Since the allocated result is greater than $15 \mathrm{~min}$ (the agreement stipulates that the MTTR of a certain shooter seat is 15 minutes), the allocated result needs to be adjusted again.
4.3. A Multiobjective Comprehensive Trade-Off Model. A certain shooter seat consists of a seat body, a seat cover cloth, and a safety belt. The agreement stipulates that the MTTR of a certain shooter seat is 15 minutes, the service life of the seat body is 12,000 flight hours, and the seat cloth is used. For 400 flight hours, the seat belt is used for 2,000 flight hours. Assume that a certain shooter seat has a lifecycle maintenance expense of 10,000 yuan and a downtime loss of 1,000 yuan per hour. Based on the assumptions proposed in the model establishment, referring to the actual data in the maintenance of a certain shooter seat, the basic parameters of the model are shown in Table 5.

The $k_{i}$ and $\alpha_{i}$ in Table 5 are the relevant expense parameters of the maintenance design, which are mainly obtained by considering the maintenance complexity parameters such as the structural complexity and accessibility of the maintenance unit; $\lambda_{i}$ is the failure rate of the $i$-th maintenance unit, which is mainly obtained according to the "Nonelectronic Component Reliability Manual" and the data accumulated in the project; and $C_{u i}$ is the unit time cost for the repair maintenance of the $i$-th repair unit. $M_{i}^{l}$ and $M_{i}^{u}$ are the maximum and minimum repair time allowed for the maintenance of the $i$-th maintenance unit at the base level, and all of them are repaired by similar products in the field. Statistics are obtained.

4.4. Results Analysis of Maintenance Allocation Method by iNSGA-II Algorithm. The algorithm development platform of this experiment is Intel(R) Core(TM) i3-4170 CPU, Windows 7, and MATLAB 13.0. The abscissa represents the objective function value of the MTTR of the system. The unit is minute $(\mathrm{min})$, and the ordinate represents the lifecycle maintenance-related expenses. The unit is thousands (K). The distribution of the Pareto solution in the evolution process is shown in Figure 3.

It can be seen from Figure 3 that the distribution of Pareto solutions before the optimization is disordered. After optimization, the Pareto solution converges to the optimal feasible solution, and with the advancement of evolution, the number of Pareto solutions is increasing. The iNSGA-II is more accurate than the NSGA-II algorithm optimization. At the same time, as the program progresses, the algorithm converges. The Pareto solution has better diversity and uniform distribution.

In addition, this article takes the basic parameters of a certain shooter seat maintenance as an example and gives the solution set that iNSGA-II performs best on these parameters as shown in Figure 4.

It can be seen from Figure 4 that both the optimal solution and the suboptimal solution are dominated by Paretodominated solutions, which further illustrates that the assumption that some excellent results proposed in this paper are approximate solutions is valid, and the iNSGA-II algorithm can effectively find these potential solutions.

The obtained Pareto solution set is shown in Table 6, and the first layer of practical feasible solutions is selected from these Pareto solutions to determine the maintainability allocation scheme. 


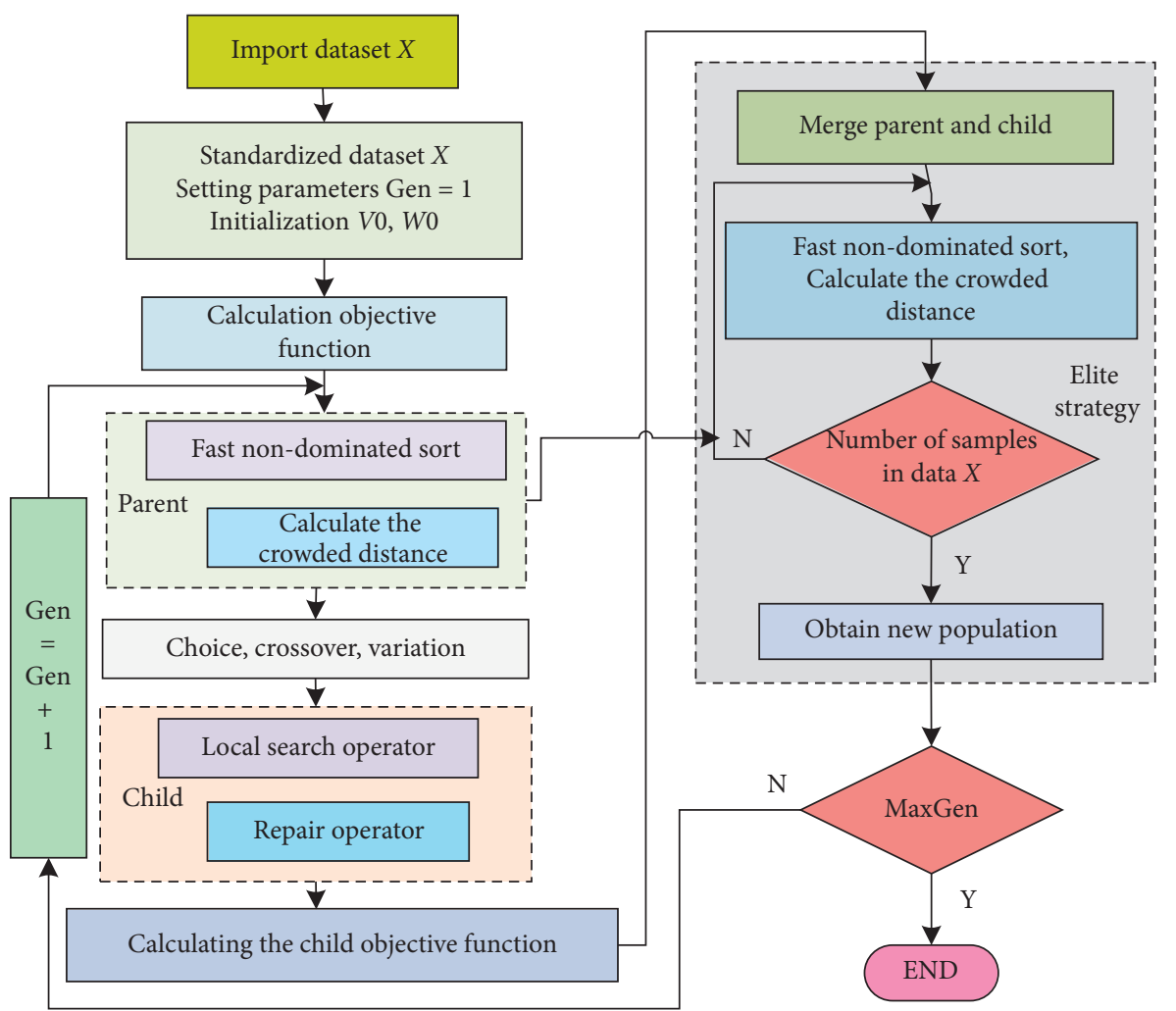

Figure 1: Improved NSGA-II flow chart.

TABLE 2: The result of the maintainability allocation of a certain shooter seat.

\begin{tabular}{lcccccccccc}
\hline LRU name & $k_{i 1}$ & $k_{i 2}$ & $k_{i 3}$ & $k_{i 4}$ & $\lambda i\left(\times 10^{-6} / \mathrm{h}\right)$ & $\bar{\lambda}\left(\times 10^{-6} / \mathrm{h}\right)$ & $k_{i}$ & $\bar{k}$ & $\beta_{i}$ & Single LRU allocation result, $J_{\text {cct }}$ \\
\hline Seat body & 10 & 4 & 4 & 1 & 28.9650 & 15.3 & 19 & 16.7 & 0.6000 & 9.0000 \\
Seat cover & 10 & 1 & 2 & 1 & 1.2996 & 15.3 & 14 & 16.7 & 9.8700 & 148.1000 \\
Seat belt & 10 & 2 & 4 & 1 & 15.5520 & 15.3 & 17 & 16.7 & 1.0000 & 15.0000 \\
\hline
\end{tabular}

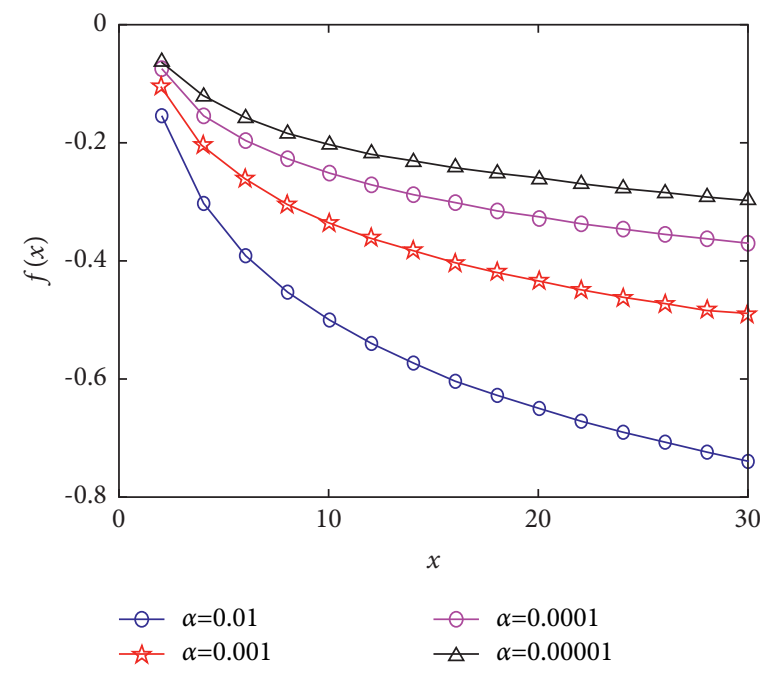

Figure 2: $f(x)=\log a(\mathrm{x}), 0<a<1$ change trend chart.

TABLE 3: The value principle of $a$

\begin{tabular}{lccccccc}
\hline $\mathrm{L}$ & $\alpha$ & $L$ & $\alpha$ & $L$ & $\alpha$ & $L$ & $\alpha$ \\
\hline $1 \leq L<10$ & 0.01 & $10 \leq L<100$ & 0.001 & $100 \leq L<1,000$ & 0.0001 & $1,000 \leq L<10,000$ & 0.00001 \\
\hline
\end{tabular}


TABLE 4: The maintainability allocation results of each LRU of a certain shooter seat.

\begin{tabular}{lccccccc}
\hline LRU name & $\lambda_{i}(\times 10-6 / \mathrm{h})$ & $\bar{\lambda}\left(\times 10^{-6} / \mathrm{h}\right)$ & $\alpha$ & $k_{i}$ & $\bar{k}$ & $\beta_{i}$ \\
\hline Seat body & 28.9650 & 15.3 & 0.001 & 19 & 16.7 & 1.2000 \\
Seat cover & 1.2996 & 15.3 & 0.001 & 14 & 16.7 & 0.5000 & 18.0000 \\
Seat belt & 15.5520 & 15.3 & 0.001 & 17 & 16.7 & 1.0000 \\
\hline
\end{tabular}

TABLE 5: Basic parameters of a certain shooter seat maintenance.

\begin{tabular}{lccccccc}
\hline LRU name & $T_{i}$ & $k_{i}$ & $\lambda_{i}\left(10^{-6} / \mathrm{h}\right)$ & $\alpha_{\mathrm{i}}$ & $C_{u i}$ & $M_{i}^{l}(\min )$ & $M_{i}^{u}(\min )$ \\
\hline Seat body & 12,000 & 5 & 28.965 & 1.3 & 600 & 15 & 3 \\
Seat cover & 400 & 3 & 1.2996 & 0.8 & 300 & 4 \\
Seat belt & 2,000 & 8 & 15.552 & 2.0 & 1,000 & 12 \\
\hline
\end{tabular}

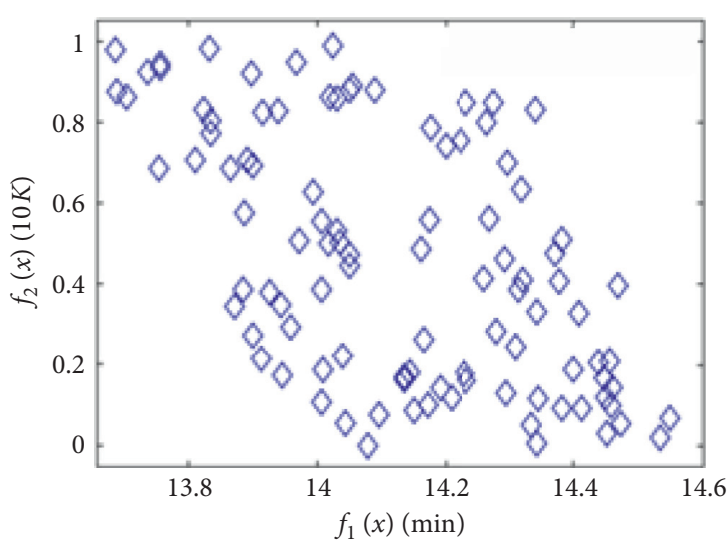

$\diamond$ Pareto solution

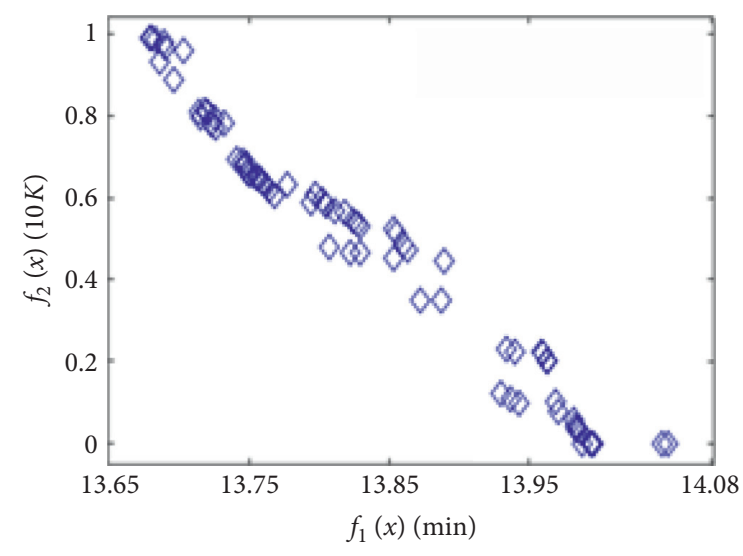

$\diamond$ Pareto solution

(a)

(b)

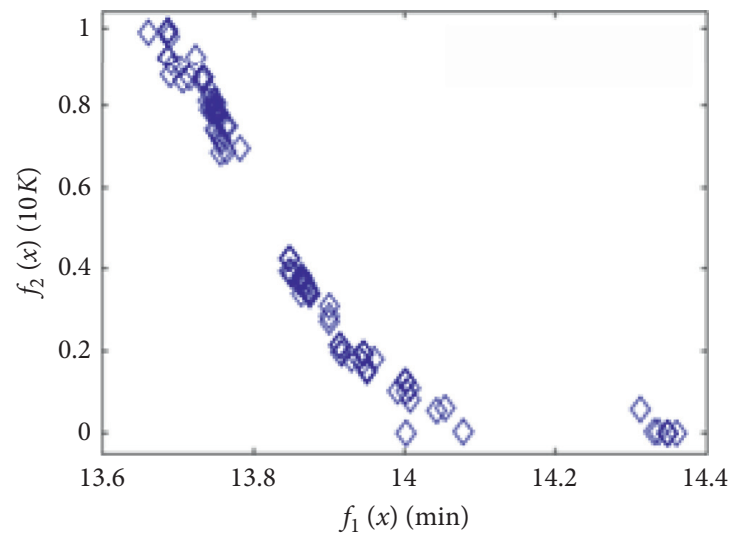

$\diamond$ Pareto solution

(c)

FIGURE 3: (a)Before optimization, (b) NSGA-II optimization, and (c) iNSGA-II optimization.

By comprehensively weighing the relationship between the lifecycle maintenance-related expenses and the MTTR, the resulting maintainability allocation scheme can provide a basis for the maintenance designer to develop or improve the design scheme. At the beginning of product design, the trade-off considers the relationship between time and expense, avoids the blindness of maintenance structure design, and improves the maintenance design efficiency. 


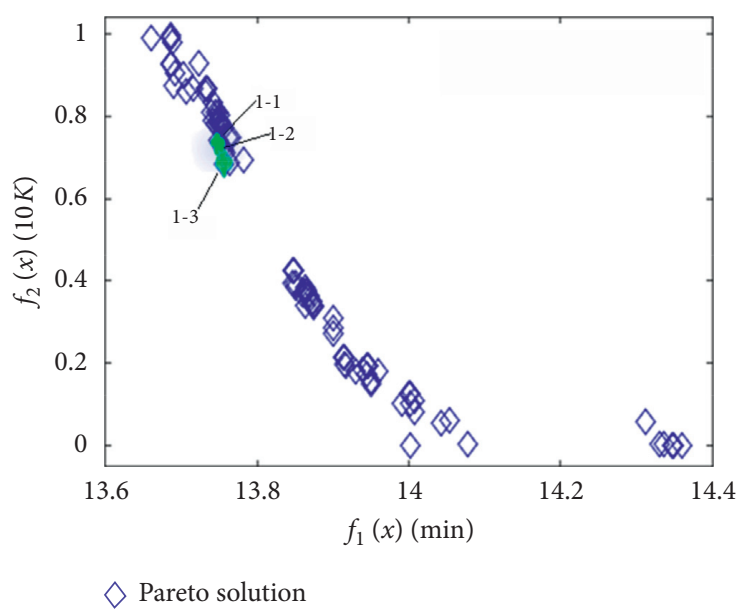

FIgURe 4: Distribution of iNSGA-II's “1-1," “1-2," and “1-3" solutions.

TABLE 6: Pareto solution set.

\begin{tabular}{|c|c|c|c|c|c|c|c|}
\hline Number of iterations & Seat body & $\begin{array}{c}\quad M_{i} \\
\text { Seat cover }\end{array}$ & Seat belt & $J_{1}$ & $J_{2}$ & Nondominated sort & Crowded distance \\
\hline 1 & 15.0015 & 3.2359 & 12.1923 & 0.7924 & 13.7142 & 1 & 0.0495 \\
\hline 2 & 15.0015 & 3.3248 & 12.0172 & 0.9900 & 13.6573 & 1 & Inf \\
\hline 3 & 15.0015 & 3.0973 & 12.4160 & 0.5401 & 13.7862 & 1 & 0.0885 \\
\hline 4 & 15.0015 & 3.0661 & 12.4162 & 0.6136 & 13.7854 & 1 & 0.1369 \\
\hline 5 & 15.0506 & 3.0679 & 12.7139 & 0.0321 & 13.9175 & 1 & 0.0413 \\
\hline$\ldots$ & $\ldots$ & $\ldots$ & $\ldots$ & $\ldots$ & $\ldots$ & $\ldots$ & $\ldots$ \\
\hline 498 & 15.6632 & 3.7359 & 12.9385 & $3.9170 e-6$ & 14.4000 & 6 & $1.8079 e-4$ \\
\hline 499 & 15.6632 & 3.7340 & 12.9385 & $4.6394 e-6$ & 14.4000 & 6 & $1.1452 e-4$ \\
\hline 500 & 15.6632 & 3.7280 & 12.9385 & $6.8677 e-6$ & 14.3998 & 6 & $9.3235 e-5$ \\
\hline
\end{tabular}

TABLE 7: Experiment record form.

\begin{tabular}{|c|c|c|c|c|c|c|}
\hline \multirow[b]{2}{*}{ Pilot projects } & \multicolumn{3}{|c|}{ Time-consuming operation } & \multicolumn{2}{|c|}{ Operator } & \multirow[b]{2}{*}{ Required tools } \\
\hline & Disassembly & Installation & Adjustment & Name & $\begin{array}{l}\text { Whether } \\
\text { proficient }\end{array}$ & \\
\hline $\begin{array}{l}\text { Certain } \\
\text { shooter seat }\end{array}$ & $55 \mathrm{~s}$ & $12 \mathrm{~s}$ & & A, B & & \\
\hline Seat body & $\begin{array}{c}3 \min 18 \mathrm{~s} \text { (two people) }+2 \mathrm{~min} \\
3 \mathrm{~s} \text { (one people) }\end{array}$ & $\begin{array}{c}4 \mathrm{~min} 4 \mathrm{~s} \text { (two people) }+3 \mathrm{~min} \\
9 \mathrm{~s} \text { (one people) }\end{array}$ & $\begin{array}{l}15 \mathrm{~s} \text { (one } \\
\text { people) }\end{array}$ & A, B & Yes & $\begin{array}{l}\text { Slotted, } 10 \mathrm{~mm} \\
\text { wrench }\end{array}$ \\
\hline Seat cover & $2 \min 3 s$ & $3 \min 9 s$ & & A & & \\
\hline Seat belt & $3 \mathrm{~min} 30 \mathrm{~s}$ (two people) & $4 \min 19 \mathrm{~s}$ (two people) & $\begin{array}{l}15 \mathrm{~s} \text { (one } \\
\text { people) }\end{array}$ & A, B & & \\
\hline
\end{tabular}

4.5. Results Verification of Maintainability Allocation Method for Multiobjective Comprehensive Trade-Off Model. In order to verify the rationality of the maintainability allocation results obtained by using the iNSGA-II algorithm for the multiobjective comprehensive trade-off model, a maintenance demonstration experiment was carried out according to the established maintenance demonstration experiment task book for a certain shooter seat, and relevant data were recorded (see Table 7 for experiment data).
The time conversion coefficient $(K)$ is introduced for the different work efficiency of general operators and proficient operators. The time conversion coefficient $(K)$ is shown in Table 8.

A certain shooter seat is operated by two general operators during field maintenance. The test data in Table 6 are converted according to the requirements in Table 7 . The conversion results are shown in Table 9.

The MTTR of a certain shooter seat is calculated according to the test data in Table 9 as follows: 
TABLE 8: Time conversion coefficient $(K)$.

\begin{tabular}{lr}
\hline Operator mix and proficiency & $K$ \\
\hline One general operator & 0.7 \\
One proficient operator & 1.0 \\
One general operator and one proficient operator & 1.7 \\
Two general operators & 1.4 \\
Two proficient operators & 2.0 \\
\hline
\end{tabular}

TABLE 9: Maintainability data sheet for a certain shooter seat.

\begin{tabular}{lccc}
\hline Maintenance project & One proficient operator need repair time (min) & Two general operators need repair time (min) & Remark \\
\hline Seat body & 22.42 & 16.01 & 3.71 \\
Seat cover & 5.2 & 12.94 & $*$ \\
Seat belt & 18.12 & $*$
\end{tabular}

Note. ${ }^{*}$ indicates that the time for the test project includes the time for the disassembly and installation of a certain shooter seat in the helicopter.

$$
\begin{aligned}
J & =\sum_{i=1}^{n} \frac{\lambda_{i} \times \bar{M}_{i}}{\sum_{i=1}^{n} \lambda_{i}} \\
& =\frac{(28.965 \times 16.01+1.2996 \times 3.71+15.552 \times 12.94) \times 10^{-6}}{(28.965+1.2996+15.552) \times 10^{-6}}
\end{aligned}
$$$$
=14.6190(\mathrm{~min}) \text {. }
$$

The MTTR of a certain shooter seat is calculated to be $14.6190 \mathrm{~min}$, which satisfies the maintainability requirements specified in the agreement; then the data in Table 9 are valid.

\section{Conclusions}

Aiming at the problem that the common maintainability allocation method does not consider the lifecycle maintenance-related expenses, a mathematical model of multiobjective optimization is established, which effectively avoids the blindness of system design and achieves the specified maintainability goal. At the same time, the iNSGA-II algorithm was used to optimize the established multiobjective comprehensive trade-off model, obtain the optimal Pareto solution, and obtain a reasonable maintainability allocation scheme for a certain shooter seat. In the experiment, the experimental results show that the multiobjective comprehensive trade-off model of total lifecycle expense and MTTR for a certain shooter seat is better than the two maintenance allocation methods. The iNSGA-II algorithm is more accurate and has a better convergence effect. The Pareto solution has better diversity and a more uniform distribution. Through the obtained Pareto solution set, the maintainability allocation scheme is finally determined.

\section{Data Availability}

The data used to support the findings of this study were supplied by the corresponding author under license and so cannot be made freely available. Requests for access to these data should be made to the corresponding author.

\section{Conflicts of Interest}

The authors declare that they have no conflicts of interest.

\section{Authors' Contributions}

Liying Jin was responsible for funding acquisition; Wensheng Wang was responsible for software; Chenxing Liang and Xuemei Ma supervised the study; and Qiang Ma and HouYong Shu validated the data.

\section{Acknowledgments}

This work was supported by the Key Research and Development Program of Hubei Province (Grant no. 2021BID001) and the Research on Multiple Regression and Fitting Technology of Simulation Data for Dynamic Umbrella Opening of Lifesaving Umbrella (Grant no. HX2021157).

\section{References}

[1] X. Xu and X. Zhu, Maintainability Allocation and Estimation Manual (gjb/z 57-1994), National Defense Science and Technology Industry Commission, Beijing, China, 1994.

[2] B. Guo, M. Wang, and H. Wu, Civil Aircraft Maintainability Engineering, aviation industry press, Beijing, China, 2018.

[3] S. Guo, Study on the Allocation Method for the Power-Shift Steering Transmission on Balance of Reliability \& Maintainability Index with the goal of Availability, Beijing Institute of Technology, Beijing, China, 2016.

[4] Q. Yan, J. Lv, and W. Liu, "Application of spares engineering based on the research method of maintainability engineering," Civil Aircraft Design \& Research, vol. 116, pp. 99-104, 2015.

[5] Q. Yan and G. Xing, "Research on the preparation of initial recommended spare parts list based on the spares support engineering," Civil Aircraft Design \& Research, vol. 3, pp. 46-53, 2018.

[6] J. Changfa and P. Jiang, "Research on maintenance engineering analysis technology based on S3000L," Aviation Maintenance \& Engineering, vol. 9, pp. 64-69, 2019.

[7] M. Gan, Maintainability Design and Verification, National Defense Industry Press, Beijing, China, 1995. 
[8] M. Gan, Military Equipment Maintenance Engineering, pp. 49-51, National Defense Industry Press, Beijing, China, 2005.

[9] X. Zhao, Maintainability Allocation of Machining Center Based on Fault Correlation, Jilin University, Changchun, China, 2018.

[10] A. Got, A. Moussaoui, and D. Zouache, "A guided population archive whale optimization algorithm for solving multiobjective optimization problems," Expert Systems with Applications, vol. 141, pp. 1-15, 2020.

[11] J. Bao, Z. Li, and Y. Zhou, "Multi-objective operation scheduling optimization of shipborne-equipment based on genetic algorithm," Journal of System Simulation, vol. 31, no. 5, pp. 901-908, 2019.

[12] Z. Lei, W. Su, and Q. Hu, "Multimode decomposition and wavelet threshold denoising of mold level based on mutual information entropy," Entropy, vol. 21, no. 2, 2019.

[13] Z. Lei and W. Su, "Mold level predict of continuous casting using hybrid EMD-SVR-GA algorithm," Processces, vol. 7, no. 3, 2019.

[14] L. Jin, X. Zhi, and S. Zhao, "Enhanced subspace clustering through combining minkowski distance and cosine dissimilarity," Journal of Intelligent and Fuzzy Systems, vol. 35, no. 5, pp. 5541-5556, 2018.

[15] L. Jin, S. Zhao, C. Zhang, G. Wei, D. Yao, and L. Mengkang, "Adaptive soft subspace clustering combining within-cluster and between-cluster information," Journal of Intelligent and Fuzzy Systems, 2019.

[16] K. Deb, A. Pratap, and S. Agarwal, T. A. M. T. Meyarivan, A fast and elitist multiobjective genetic algorithm: NSGA-II," IEEE Transactions on Evolutionary Computation, vol. 6, no. 2, pp. 182-197, 2002.

[17] X. Hu, J. Zhuang, and D. Yu, "Multi-objective unsupervised feature selection algorithm utilizing redundancy measure and negative epsilon-dominance for fault diagnosis," Neurocomputing, vol. 146, pp. 113-124, 2014.

[18] L. Jin, S. Zhao, W. Du, and Y. Xuesong, "Research on multiobjective optimization of switched flux motor based on improved NSGA-II algorithm," Journal Of Process Mechanical Engineering, vol. 233, no. 6, pp. 1268-1279, 2019. 